\title{
Barreras para el comportamiento saludable desde la perspectiva de los determinantes sociales de la salud-enfermedad
}

Barriers to healthy behavior from the perspective of the social determinants of health and disease

\author{
Daniel Pinzón Gómez ${ }^{1}$
}

\begin{abstract}
RESUMEN
El propósito del presente artículo es hacer un análisis crítico al planteamiento epidemiológico clásico dominante que explica la salud-enfermedad desde la genética y los estilos de vida. Se plantea cómo desde los determinantes sociales de la salud, relacionados con las inequidades sanitarias, están fuertemente influenciados por la estructura política, económica, cultural, ambiental y demográfica que pueden plantear barreras para la adopción de un estilo de vida saludable, especialmente a personas en condiciones de pobreza extrema, conflicto armado, desastres y demás adversidades, que son circunstancias dominantes en los países de bajos y medianos ingresos. Se discute la necesidad de trascender el paradigma de educación para la salud, el cual es altamente prescriptivo y no ofrece los resultados que se esperan. La investigación es de carácter documental, se basó en una revisión bibliohemerográfica. Se concluye que es necesario trascender hacia un paradigma holístico, donde se explique que el proceso salud-enfermedad y en el cual se hace necesario trabajar en la solución de las inequidades sanitarias estructurales que permitan resolver las condiciones de vida que hacen que las personas persistan en los hábitos que acarrean mala salud.
\end{abstract}

Palabras clave: Determinantes sociales, estilo de vida, proceso salud-enfermedad, barreras para el comportamiento saludable, inequidades en salud.

\begin{abstract}
The purpose of the present article is to make a critical analysis to the dominant classic epidemiological position that explains health-disease duality from genetics and lifestyles. It talks about how political, economic, cultural, environmental and demographic structure are strongly influenced by the social determinant of health, related to sanitary inequities, which may pose barriers to the adoption of healthy lifestyles, especially to people in extreme poverty, armed conflict, disasters and other hard conditions, which are prevailing circumstances in low and middle income countries. It discusses the need to transcend the paradigm of health education which is highly prescriptive and does not offer the expected results. The research is documentary, and was based on a journalistic literature review. It concludes that it is necessary to transcend into a holistic paradigm to explain the healthdisease process; it is also essential to work in the solution of the structural sanitary inequities to solve living conditions that make people to persist in habits that carry bad health.
\end{abstract}

Keywords: Social determinants, lifestyles, health-disease process, barriers to healthy behavior, health inequities.

${ }^{1}$ Master of Public Health [C] Atlantic International University [EE.UU.]. Administrador en Salud, Gestión Sanitaria y Ambiental (AS GSA). Tp. en atención prehospitalaria de la Universidad de Antioquía. Docente en la Universidad Adventista de Colombia. Coinvestigador (Grupo de Epidemiología) Facultad Nacional de Salud Pública Colombia.

Correspondencia:pinzon.gomez@gmail.com 


\section{INTRODUCCIÓN}

El sector científico y académico adventista viene abogando por una comprensión holística de la salud que abarque las dimensiones biológica, psíquica, espiritual y social. (Lechleitner, 2013). El mayor desarrollo en relación con la salud ha estado en el plano biológico, gracias al gran avance de las ciencias biomédicas; en menor desarrollo, pero no menos importante, las ciencias del comportamiento vienen realizando aportes sustanciales a la psicología de la salud; en cuanto a lo espiritual, se logró su inclusión a nivel nacional, pero su desarrollo epistemológico es invariablemente escaso, con unas poquísimas aportaciones de carácter científico-práctico; finalmente lo social de la salud, aunque vindicado en el discurso, ha sido prácticamente marginado debido al protagonismo de lo biomédico que lo ha ensombrecido (Contandriopoulos, 2006).

No obstante, el tránsito de la era de las enfermedades crónicas y su paradigma de factores de riesgo (caja negra), así como la necesidad de encontrar explicaciones más allá del individuo al problema salud-enfermedad, han obligado a la epidemiología clásica a migrar hacia la ecoepidemiología (paradigma de cajas chinas) (Villa, Altamirano, \& García, 2012) (Ariza, López, Martínez, \& Arias, 2004) y su consabida epidemiología social (Álvarez L. S., 2009), (OPS, 2010a) en la cual se introduce a la teoría de la producción social de la enfermedad que plantea la existencia de condicionantes políticos, económicos, sociales, culturales, ambientales, demográficos, entre otros, en un contexto de salud internacional (Pulgarín, 2010) como circunstancias que explican, de manera holística, la determinación del proceso salud-enfermedad (PSE).

En este artículo se expone la perspectiva de los determinantes (condicionantes) sociales de la salud (OMS, 2009), (Peñaranda, y otros, 2011), como parte de la iniciativa que la Organización Mundial de la Salud (OMS) viene analizando desde el 2005, con el fin de hacer una aproximación holística y contextual a la tradicional propuesta por la epidemiología clásica causalística. Aborda el enfoque de estilo de vida saludable (EVS) desde una perspectiva de la determinación social del PSE en el que se hace un análisis crítico al paradigma tradicional que transfiere la resposabilidad de la salud-enfermedad al plano de las elecciones individuales, explicando cómo las inequidades sanitarias presentan barreras para la adopción de hábitos sanos (WHO, 2005a); igualmente expone la necesidad de trascender en las estrategias de educación para la salud (EpS) para el cambio hacia el comportamiento saludable que, si bien pueden resultar en un activo positivo, se quedan en el plano prescriptivo, sin lograr incidir en los fenémenos estructurales, reduciéndose a recomendaciones que no logran transformaciones sustanciales, entonces, ¿qué significado tiene promover estrategias educativas para el cambio de hábitos insalubres que no resuelven las condiciones de vida haciendo que las personas persistan en ellos? De esta manera se plantea la necesidad de ir más allá de las propuestas de EpS restringidas la plano de las decisiones personales y trabajar en la comprensión que el comportamiento sano se explica desde la corresponsabilidad que más que un asunto individual, es un asunto social y político.

\section{Lo social de la salud}

Desde la perspectiva filosófica, parece existir un consenso en aceptar la indivisibilidad de la persona humana, en tanto dimensión ontológica, en el que tradicionalmente se ha considerado la composición biológica, síquica y social del individuo (Contandriopoulos, 2006), situación que llevó, en el campo de la salud, al desarrollo del modelo biopsicosocial propuesto por Engel (MontanerGomiz y colaboradores, 2012). No obstante, el "vacío espiritual" sentido a partir de la "inseguridad psicológica" permite identificar a Toynbee, (Khayat, 1998) una cuarta dimensión: la espiritual. Es así como la OMS aprueba, en 1983, la histórica resolución WHA37.13, donde se reconoce a la dimensión espiritual, posteriormente, en 1998, un grupo especial del Comité Ejecutivo revisor de la Constitución, por medio de la resolución EB 101.R2 1998, recomendó a la Asamblea modificar la definición de salud desarrollada en 1946: "La salud es un estado de completo bienestar físico, mental, social y espiritual, y no solamente ausencia de dolencia o enfermedad". Dicha propuesta aún no ha recibido sanción (JiménezEspinoza, 2012).

De estas subdimensiones, la filosofía adventista de la salud ha reconocido tres de ellas: la física (biológica), la mental (síquica) y la espiritual, identificándose en la literatura denominacional un distanciamiento frente a la dimensión social de la salud, hasta ser invisibilizada. Esfuerzos planteados por Muñoz-Olivarez (2010) defienden la composición bio-psico-socio-espiritual y más recientemente, en la Cumbre de Salud de Orlando, se identifica un esfuerzo por integrarla (Lechleitner, 2013), donde lo socialsanitario se entrevé en tanto el individuo influencia al colectivo, a través del "servicio comunitario," más que una influencia recíproca inherente.

Estos abordajes, desde la interpretación y la práctica de la salud adventista, han estado desde 
sus inicios dominados por una teología de la salud medicalizante, la cual ha seguido el discurso positivista, predominando los argumentos que sostienen que la salud puede ser interpretada desde lo clínico-individual, la investigación biomédica basada en la evidencia y un "simple cambio" de estilo de vida (Lechleitner, 2013), desconociendo los abordajes de la investigación histórico-hemenéutica y crítico-social de la salud colectiva, la medicina social, la ecoepidemiología que han puesto en crisis al paradigma epidemiológico clásico de las causas próximas de la enfermedad, como determinantes de la salud (Benach J. 2012a), (Montaner-Gomis I y colaboradores, 2012), en tanto existen explicaciones al PSE como las planteadas por Marmot (2007) al indagar por las "causas de las causas" o "causas estructurales" de la enfermedad; las interpretaciones formuladas por Breilh (2003) al abogar por una explicación desde la "determinación social del proceso saludenferemedad" o la de los condicionantes sociales de la salud, propuesta por Peñaranda (2011), la cual plantea que un ser ético nunca podrá ser determinado, siendo estas últimos perspectivas planteadas desde un mayor rigor epistemológico.

De igual manera, estas aproximaciones sociales a la salud pueden explicar, entre otras, las barreras que existen para que las personas tengan un comportamiento sano, tema de frontera en salud pública hoy, y prioritario en la agenda reciente de la OMS (OMS, 2009).

\section{Determinantes sociales de la salud (DSS)}

Uno de los modelos más aceptados y comunmente usados para explicar la determinación de la salud es el propuesto por H. Lamfraboise y M. Lalonde denominado "Campos de Salud" (Lalonde, 1974), donde se explica que la salud de los canadienses estaría condicionada por la biología, el ambiente, los estilos de vida y los servicios de salud. Este modelo pone un punto de quiebre a la tradicional explicación unicausal y la triada ecológica de la enfermedad, para adentrarse en un paradigma multicausal. Seguidamente, Dever (1976) pudo cuantificar la carga de cada una de estas variables sobre la población estadounidense.

Sin embargo, este modelo se agota en la focalización de la promoción de la salud en los estilos de vida, al considerarlos como una decisión individual con el efecto negativo de medicalizar la prevención, sin tener una repercusión significativa en los comportamientos de salud de las personas, igualmente descuida la dimensión social de la salud (Cofiño R., 2012)
Paralelamente, el desarrollo de la epidemiología social ha mantenido sus contribuciones a la interpretación de la salud, entre las que se reconocen la declaración de Alma Ata de 1978 sobre Atención Primaria en Salud, la Primera Conferencia de Promoción de la Salud, conocida como la Carta de Ottawa en 1986 (OMS,1986) y sus sucesivas conferencias que han tenido aportes como las Dahlgren y Whitehead (WHO, 2005b), quienes difundieron el modelo de arcoíris, uno de los más conocidos en determinación social de la salud; igualmente el reconocido G. Rose (1991), Diderichen y colaboradores, Mackenbach y colaboradores, Brunner, Marmot y Wilkinson (WHO, 2005b). En Latinoamérica y el Caribe (LAC) el desarrollo de la medicina social, la epidemiología crítica (Breilh, 2003) y la salud colectiva (Almeida-Filho,2000) han constribuido significativamente a la consolidación de un pensamiento social en la salud, con una mirada holística e integradora al enfoque biomédico reduccionista morbicéntrico.

En el 2005, la OMS estableció la Comisión sobre Determinantes Sociales de la Salud (CDSS), con el fin de acopiar la evidencia científica que permitiera identificar la participación de los procesos sociales en el aporte a la mala salud de las poblaciones, retomando estudios desarrollados desde los años 70 por varios investigadores, especialmente por la corriente de la epidemiología social (WHO, 2005a).

La OMS los DSS, como "las circunstancias en que las personas nacen, crecen, viven, trabajan y envejecen, incluido el sistema de salud". (WHO, 2005b).

Circunstancias que estarían configuradas por un conjunto más amplio de fuerzas (económicas, sociales y políticas) y que explican la mayor parte de las iniquidades en salud, esto es, de las diferencias injustas y evitables observadas. (Montaner-Gomis, 2012).

El aspecto clave, central y problemático analizado por los DSS son las inequidades en salud (Álvarez, 2012) que van más allá de la tradicional, dominante e imprecisa explicación científica que el problema de la enfermedad es lo biológico-genético y el fracaso moral de la conducta del individuo (Benach, 2012a). Las explicaciones sociológicas detallan que el PSE se distribuye de manera desigual a través de las clases y estratos sociales. A medida que se analizan las diversas clases sociales, la salud comienza a presentar unas variaciones y unos gradientes, demostrando que a menor clase social, menos posibilidades de empleabilidad digna, menos educación, menores 
ingresos; igualmente estos se relacionan de manera directa con la etnia y el género; asimismo, esta relación en la clase social coincide con la distribución de una mayor mortalidad, menor esperanza de vida al nacer, menor peso al nacer, mayor predisposición de enfermar y de morir entre las personas en desventaja social (OMS, 2005); asimismo, se ha podido evidenciar que a medida que se desciende en la escala social, las personas tienden a tener mayor propensión a las adicciones de todo tipo (Benach J., 2012b), también se expresan mayores focos de violencia, accidentalidad y lesiones en general (Peden M y colaboradores, 2004). En este sentido, la pobreza engendra mala salud, por lo que los pobres mueren más jóvenes, sufren más discapacidades y están expuestos a mayores riesgos como consecuencia de las condiciones insalubres que se escapan de su posibilidad de controlar (Rodríguez, 2003). Este fenómeno se explica a través de la configuración de determinantes intermedios y estructurales de la salud, expresados en el marco conceptual de DSS.

\section{El mito de la pistola cargada y el dedo sobre el gatillo}

Las líneas más extremas del positivismo biomédico de la salud clásica han llevado a defender ideas, que si bien pueden tener evidencia científica - tal como lo demostró Lalonde y Dever - han constribuido a posicionar más una ideología política de corte neoclásico, que un hecho científico, en la que se insiste que la salud es un bien privado regido por las leyes del mercado (Hernández, 2003), que depende de la biología y los genes (determinismo biológico) y los estilos de vida - EV, elecciones personales, en los que si la persona pierde la salud, debe pagar por su descuido (Benach \& Montaner, 2005) -. Este binomio ejemplificado con una ruleta rusa, donde la genética se compara a un suicida con una pistola cargada y el estilo de vida corresponde a tener el dedo sobre el gatillo, en tanto depende de la elección personal el disparar o no, es decir, la activación de la enfermedad por motivos genéticos dependerá del comportamiento y los hábitos individuales. Esta metáfora, lejos de demostrar el complejo integral del PSE es culpabilizante, reduccionista y simplista; desconoce las circunstancias contextuales y estructurales y las verdaderas "causas de las causas de la enfermedad" que pueden ser analizadas siguiendo con la alegoría, tratando de responder a preguntas cómo ¿y quién fabricó la pistola?, ¿cómo llegó la pistola a manos del suicida?, ¿qué circunstancias hacen que la persona porte el arma y llegue a tener conductas suicidas?

La OMS define el estilo de vida (EV) como "forma general de vida basada en patrones de comportamiento identificables, determinados por la interacción entre características personales individuales, las interacciones sociales y las condiciones de vida socioeconómicas y ambientales", (Fenández y otros, 2009).

Esta definición tiene sus orígenes en lo que $\mathrm{M}$. Weber plantea a fines del siglo XIX. Él argumenta que el EV está dado por la conducta individual (elección) determinada a su vez por las posibilidades de vida (oportunidades) (Cockerham, 2012), en este sentido determinadas formas de comportamiento dependerán del contexto en el que los individuos se desarrollen y pueden estar limitadas o ampliadas por factores ambientales o sociales. A su vez, las pautas de conducta de un grupo están determinadas socialmente, lo que se refleja en su EV. Muchas de estas pautas se aprenden en la relación con los pares, los padres, la familia, los medios de comunicación y en la escuela, sin embargo, estas están sujetas a modificaciones de acuerdo con las situaciones sociales que se presenten (Vargas, 2010).

Dado que el EVS ha venido a ocupar un espacio predominante en la comprensión del PSE, su concepto y contexto, tal como se ha descrito, permite ver que existen tanto en la literatura científica como en el discurso biomédico tradicional y denominacional una tendencia que se expresa en: a) énfasis absoluto en la elecciones individuales y la conducta; b) marginalidad de un análisis contextual que determinan las conductas; c) ausencia en la comprensión integral de que el EVS es un asunto de corresponsabilidad entre el individuo y la sociedad.

De esta manera, el énfasis en que lo genético está condicionado por las elecciones personales busca ensombrecer la posibilidad de mirar un contexto más amplio de determinación, en tanto que la interpretación clásica es de causalidad. La explicación causal del PSE libra de responsabilidad a los determinadores, la explicación contextual es abarcante y supone la necesidad de trabajar no solo en las causas próximas, sino en las causas estructurales. Mirado de este modo, la salud deja de ser una asunto médico para convertirse en una cuestión política.

De esta manera, el desafío no solo es, por ejemplo, en propender que las personas con problemas cardiovasculares cambien de hábitos a través de la educación y la información, es también trabajar en procura de que hayan entornos saludables (escenarios deportivos adecuados y seguros, tiendas que expendan alimentos sanos, entre otros), es cabildear por el control y regulación de quienes, en aras del dinamismo de la economía, tienen inmersa la sociedad en la cultura de las golosinas y el "snack" chatarra al expender en toda clase de 
tiendas alimentos y bebidas insalubres, es propiciar el desestímulo en la fabricación y venta de alcohol y tabaco. Es también que la gente tenga empleo digno, con el cual puedan acceder a una mayor variedad de oportunidades saludables. Finalmente estas acciones estructurales y contextuales no son de tipo individual, tampoco del campo biomédico, sino son de carácter social y político, esto implica que al trabajar por los EVS, es necesario desmedicalizar la prevención de la enfermedad y la promoción de la salud.

\section{Limitaciones sociales para la elección saludable}

Aunque en la actualidad muchos de los problemas de salud, propios de las sociedades desarrolladas, están siendo experiementadas en los países de bajos y medianos ingresos (PBMI), particularmente en los que respecta a enfermedades crónicas no transmisibles (ECNT), en lugar de estar dándose una transición epidemiológica, lo que realmente está ocurriendo es una acumulación epidemiológica, donde los males del mundo desarrollado aparecen en estos países sin haberse resuelto gran parte de los problemas de salud, típicas del subdesarrollo del siglo XIX (Feo, 2004).

Pese a ello, las estrategias de EpS, para el cambio de comportamiento, son trasladadas de los países de altos ingresos (PAI) a los PBMI, donde se asume que las personas tienen un nivel de vida estándar y todas pueden, de alguna forma u otra, adoptar hábitos de vida semejantes; no obstante las circuntancias en las cuales las personas viven, crecen y se desarrollan son diversas y varían entre regiones y áreas ricas y pobres, presentando estas últimas limitaciones para el desarrollo de su capacidad de elección, de allí que se demuestre que las ECNT y las adicciones son más complejas en las regiones donde prevalecen las desventajas sociales (Benach \& Montaner, 2005), (Benach, 2012b).

La propuesta que un mejor estilo de vida garantiza la salud, puede ser aplicada a contextos sociales favorables, como los que pueden existir en los PAI y clases medias altas de los PBMI, pero pueden ser marginalmente aplicados en todos los contextos de LAC, donde la probreza es prevalente y coincide con bajos niveles de escolaridad, desempleo, subempleo o empleo informal, inseguridad alimentaria, precariedad de la vivienda, dificultades de acceso al saneamiento básico, recurrencia de desastres, conflictos armados y la vulneración de derechos (acentuados en grupos sociales como niños, mujeres, comunidades afro, indígenas, ancianos y discapacitados).

Particularmente, algunas apuestas de tipo médicoeducativas para el cambio a un comportamiento saludable provenientes de EE.UU. (v.g. los programas de la Universidad de Loma Linda, Health Education Resources [HER], Florida Hospital y Weimar Center of Health \& Education, ampliamente promovidos en LAC), y que son replicadas en las escuelas de salud pública adventistas de las Américas, así como en puestos de avanzada (outpost centers) de la región, promueven las teorías del cambio, partiendo del hecho de que la capacidad de elección, alojada en el lóbulo frontal del cerebro, puede ser modelada a discreción, defendiendo la idea de que para lograr una mejor calidad y larga vida es suficiente con la adopción de "simples cambios de estilo de vida" (Lechleitner, 2013).

Las teorías de cambio conductual a partir de la elección dejan de lado la construcción más amplia del concepto de estilo de vida, considerado por M. Weber que si bien considera la "conducta de vida" del individuo en el plano electivo, también incluye las "oportunidades de vida" (Weber,1922), determinadas por las circunstancias sociales de la persona, ampliando la posibilidad de un estilo de vida saludable, las cuales "son mayores en los grupos socioeconómicos medios y altos que tienen los mejores recursos para apoyar las elecciones de estilo de vida". (Cockerham, 2012).

Los prometedores resultados del Estudio de Salud Adventista (Fraser, 2003) que demuestra que el EV adventista aumenta la longevidad (en aproximadamente 10 años) y reduce las enfermedades crónicas no transmisibles se han concentrado en PAI (Beeson L., 1999), (Beeson, Mills, Phillips, Andress, \& Fraser, 1989), donde paradógicamente los adventistas representan una minoría (Pinzón-Gómez, 2013), (AAN, c2005-2010). No resulta extraño que los mejores resultados de estos estudios se encuentren en una de las zonas más prósperas de EE.UU., a decir, Loma Linda. Sin embargo, no se trata de argumentar que la propuesta adventista sea anodina, sino que no ha sido aleatorizada en países pobres, paticularmente en LAC; igualmente, las instituciones de la región que promueven el EVS adventista, a pesar de argumentar éxitos en su implementación, especialmente en centros de vida sana (outpost centers), no han documentado las experiencias, reduciéndolas a una disertación anecdótica, situación que no permite determinar si el éxito tenido en los PAI se comporta de manera similar en los PBMI.

A la luz de la determinación social de la salud, la aleatorización del éxito del EV adventista puede resultar problemática en virtud de las cifras de iniquidad que acompañan al mundo: $42 \%$ de la población mundial sobrevive con menos de dos 
dólares al día (Finkelman, Galvao, \& Henao, 2010); $14 \%$ (alrededor de mil millones vive en condiciones de pobreza absoluta, y es este sector de la población el que crece más rápido (W.Volkheimer, Scafati, \& Melendi., 2013), por cada siete personas que hay en el mundo, una no tiene alimentos suficientes para estar saludables y llevar una vida activa. El hambre y la desnutrición son consideradas, a nivel mundial, el principal problema para la salud, más que el SIDA, la malaria y la tuberculosis juntas (DOW-URV, 2012) y no las enfermedades crónicas como ha pretendido demostrar la epidemiología clásica. Esta situación, lejos de ser ideal, representa las graves iniquidades sociales y que se expresan en las condiciones de salud del mundo, siendo un reto para la adopción de prácticas saludables tanto en adventistas como en quienes no lo son.

\section{CONCLUSIONES}

El discurso científico de la salud adventista, especialmente aquel relacionado con el Estudio de Salud Adventista y el Florida Hospital, entre otros, está fuertemente soportado en el enfoque de medicina basada en la evidencia, bajo la tradicional investigación positivista que enfatiza que una mejor calidad de vida y mayor longevidad se alcanza con la adopción de un EVS. Este enfoque está centrado en que las personas pueden cambiar sus hábitos a discreción a través de la autoconciencia, voluntad independiente y conciencia moral, la modelación de estos comportamientos puede ser desencadenada por la estrategias de EPS de corte prescriptiva.
Esta evidencia carece de contexto científico con aproximaciones constructivistas que den cuenta de la explicación del PSE, desde la determinación social de la salud en contextos de conflicto armado, desastres, sequías, inseguridad alimentaria, precariedad en saneamiento básico, pobreza, desempleo, personas en situación de dependencia (en especial niños, mujeres y ancianos) y personas con vulneración de derechos, situaciones que, sumadas en su conjunto, representan el grueso de la población mundial. Paradójicamente el éxito de estos estudios ha tenido aplicación en PAI $\mathrm{y}$, aunque se han extrapolado a PBMI, su práctica se limita a lo anecdótico. Además, desconoce las condiciones sociales relacionadas con el contexto político, social, ambiental y demográfico en el que las personas pueden estar inmersas, situación que puede resultar limitante para la adopción de hábitos saludables. Se requiere que la comunidad científica adventista avance en estudios sobre EVS triangulados que posibiliten el análisis de los hábitos de vida y salud en PBMI.

Conviene pues emprender iniciativas entusiastas, para que las instituciones adventistas de los PBMI documenten sus experiencias y emprendan estudios aleatorizados sobre mortalidad, morbilidad sentida, discapacidad y prácticas saludables en contextos de adversidad y en circunstancias de desventaja social, aplicando métodos cualitativos y cuantitativos de investigación y no limitarse a los recursos que proporciona la medicina basada en la evidencia. 


\section{REFERENCIAS BIBLIOGRÁFICAS}

Agencia Adventista de Noticias (AAN). (c2005-2010). Los adventistas ya son más de 16,6 millones en el mundo. Obtenido de Iglesia Adventista del Séptimo Día. Unión Ecuatoriana: http://adventistas.ec/noticias/ UE/2010/10_18_adventistas.php.

Dow Chemical Ibérica; Universitat Rovira i Virgili,. (2012). Módulos universitarios de desarrollo sostenible. Obtenido de DOW/URV Chair of Sustainable Development : http://www.desenvolupamentsostenible. o r g / i n d e x.p h p ? o p t i o n $=$ c o m content\&view $=$ article $\&$ id $=235 \&$ Itemid=268\&lang $=e$ s

Almeida-Filho, N. d. (2000). La ciencia tímida. Ensayos de deconstrucción de la epidemiología. Buenos Aires: Lugar Editorial.

Álvarez, L. (2012). Los estilos de vida en salud: del individuo al contexto. Revista Facultad Nacional de Salud Pública, 30(1), 95-101.

Álvarez, L. S. (2009). Los determinantes sociales de la salud: más allá de los factores de riesgo. Gerencia y Políticas de Salud, 8(17), 69-79.

Ariza, E., López, C., Martínez, O., \& Arias, S. (2004). Ecoepidemiología: el futuro posible de la epidemiología. Facultad Nacional de Salud Pública, 22(001), 139-135.

Benach, J. (9 de 10 de 2012). Joan Benach. Neoliberalismo, Determinantes sociales e inequidad en salud (1). Recuperado el 24 de Febrero de 2013, de GredsEmconet: http://www.youtube.com/watch?v=U2Bo$\mathrm{KMPeO} 8$.

Benach, J. (20 de 5 de 2012). La desigualdad en salud es una epidemia, la más grave que existe., http://www.youtube. $\mathrm{com} / \mathrm{watch} ? \mathrm{v}=\mathrm{q} Y$ TBM 2 Y SoLE\& 1 ist $=$ PLZ5d cw5E5JGQ8xOALIQGsXIEh5MWgpIK. Obtenido de Sicom TV. Solidaritat i Comunicació.

Benach, J., \& Montaner, C. (2005). Aprender a mirar la salud: cómo la desigualdad social daña nuestra salud. Maracay: Instituto de Altos Estudios en Salud Pública "Dr. Arnoldo.

Breilh, J. (2003). Epidemiología crítica. Ciencia emancipadora e interculturalidad. Buenos Aires: Lugar Editorial.

Beeson, L. (1999). The Adventist Advantage. College and University Dialogue, 11(2), 8-11.

Beeson, W., Mills, P., Phillips, R., Andress, M., \& Fraser, G. (August de 1989). Chronic Disease Among Seventh-day Adventist a Low-risk Group. Racionale, methodology and description of the population. Cancer, 598-604.

Cabrera, G. (2004). Teorías y modelos en salud pública del siglo XX. Revista Corporación Médica, 35(3), 164-168.

Cockerham, W. (2012). Medical Sociology. Boston: Prentice Hall.

Cofiño R., Pasarín MI., Segura A. (2012). ¿Cómo abordar la dimensión colectiva de la salud de las personas?
Informe SESPAS 2012. Gaceta Sanitaria, 26(S), 88-93.

Contandriopoulos, A.-P. (2006). Elementos para una "topografía" del concepto salu. Ruptures. Revista Interdisciplinaria de Salud, 11(1), 86-99.

Dever, G. (1976). An epidemiological model for health policy analisys. Social Indicators Research 2, 453-456.

Fraser, G. (2003). Diet, life expectancy and chronic disease: Studies of Seventh-Day Adventists and Other Vegetarians. New York: Oxford University.

Feo, O. (2004). La Salud Pública en los Procesos de reforma y las Funciones ESENCIALES de Salud Pública. Revista Facultad Nacional de Salud Pública, 22(99), 61-71.

Finkelman, J., Galvao, L., \& Henao, S. (2010). Gobernanza de la Salud Ambiental. En J. Finkelman, L. Galvao, $\&$ S. Henao, Determinantes ambientales y sociales de la salud (págs. 33-65). Washington: Organización Panamericana de la Salud.

Gálvez-Vivanco, C. A. (2002). Poder para cambiar. Los hábitos de salud de manera feliz y para siempre. Lima: Imprenta Unión.

García, M. (2003). Limitaciones y subterfugios de la medicina basada en la evidencia. Revista Medicina Clínica, 120(5), 197.

Health, W. H. (2005). Towards a Conceptual Framework for Analysis and Action on Social Determinats of Health. Londres: World Health Organization. Comission on Social Determinants of Health.

Hernández, M. (2003). Neoliberalismo en salud: desarrollos, supuestos y alternativas. En D. Restrepo, La falacia neoliberal. Críticas y alternativas (247-261). Bogotá D.C.: Ediciones Antropos Ltda.

Fernández- Ledesma , JD; Orrego-Ramírez, L; Martínez Mejía, CM; Uribe,L; Quintero MA; Agudelo-Viana, G; Franco-Restrepo JC.. (2009). Estudio para determinar el nivel de satisfacción de la población beneficiada de actividad física y estilos de vida saludables ofrecidos por la Secretar. Itaguí: Universidad Coopetativa de Colombia.

Jiménez-Espinoza, D. (2012). Estilos de vida saludable y medicina. Estilo de vida saludable. Tiempo de elección, tiempo de decisiones, 7-9.

Khayat, M. (1998). Spirituality in the Definition of Health The World Health Organization's Point of View. Obtenido de Swiss Academy of Medicine and Ethics: http://www.medizin-ethik.ch/publik/spirituality definition_health.htm.

King, W. (2011). Creation Health. Florida Hospital. Miami, Florida, Estados Unidos.

Lalonde, M. (1974). A New Perspective on the Health of Canadians. A working document. Ottawa: Ministty of National Health and Welfare. 
Lechleitner, E. (15 de abril de 2013). Adventist Church Promotes Next Step for Comprehensive Health Ministry. Recuperado el 4 de mayo de 2013, de Adventist News Network (ANN): http://news.adventist.org/en/archive/ articles/2013/04/15/adventist-church-promotes-nextstep-for-comprehensive-health-ministry/

Marmmot, M. (2007). Achieving health equity: from root to fair outcomes. The Lancet, 370, 1153-1163.

Muñoz-Olivarez, J. (2010). Vida y estilos de vida saludables en salud mental. Psyque-Koine, 1(1), 5-6.

Montaner-Gomiz I, Foz Gil G., Pasarín-Rúa I.. (2012). La salud: ¿un asunto individual? Actualización en Medicina de Familia, 8(7), 374-382.

Organización Mundial de la Salud. (2009). Subsana las desigualdades en una generación. Alcanzar la equidad sanitaria actuando sobre los determinates sociales de la salud. Buenos Aires: Ediciones Journal S.A.

Organización Mundial de la Salud, OMS; Salud y Bienestar Social de Canadá, SBC; Asociación Canadiense de Salud Pública, ACSP. (1986). Carta de Ottawa para la promoción de la salud. Conferencia internacional sobre promoción de la salud. Hacia un nuevo concepto de la salud pública, (6). Ottawa.

Organización Panamericana de la Salud. (2010). Presentación y marco coceptual. En: Organización Panamericana de la Salud, Módulos de principios de epidemiología para el control de enfermedades, 24. Washington: Organización Panamericana de la Salud.

Peden M, Scurfield R, Sleet D, Mohan D, Hyder A, Jarawan E, Mathers C, (edi). (2004). World Report on Road Traffic Injury Prevention. Geneve: World Health Organization.

Peñaranda, F., Pérez, E., Restrepo, M., Sarasti, D., Cáceres, F., \& Borrero., Y. (2011). ¿Determinantes? o determinación social de la salud.
Pinzón-Gómez, D. (13 de enero de 2013). Estilo de vida saludable: limitaciones del enfoque biomédico. Artículo sin publicar. Medellín, Antioquía, Colombia.

Vargas, S. (2010). Estilos de vida en la salud. En A. Abarca, M. Acuña, E. Alfaro, G. Amador, E. Androetto, \& M. Ascencio, La salud pública en Costa Rica. Estado actual, retos y perspectivas (101-124). San José: Universidad de Costa Rica. Escuela de Salud Pública.

Villa, A., Altamirano, L., \& García, G. (2012). Epidemiología $y$ estadística en salud pública. Ciudad de México: McGraw-Hill.

Weber, M. (1964). Economía y sociedad. México: Fondo de Cultura Económica.

W.Volkheimer, Scafati, L., \& Melendi., D. (17 de mayo de 2013). Población mundial. Obtenido de Breve enciclopedia del ambiente: http://www.cricyt.edu.ar/ enciclopedia/terminos/PoblacMund.htm

World Health Organization. Comission on social determinats of health. (2005). A Conceptual Framework for Action on the Social Determinants of Health. World Health Organization. Cairo: World Health Organization.

World Health Organization. Commission on Social Determinants of Health. (2009). Closing the Gap in a Generation: Health Equity Through Action on the Social Determinants of Health. Washington: World Health Organization.

World Health Organization. Comission on the Social Determinants of Health. (WHO-CSDH). (2005). Towards a Conceptual Framework for Analysis and Action on social Determinats of Helath. Geneve: World Health Organization. 